\title{
Análisis del sobrepeso, obesidad, actividad física y autoestima de la niñez en el área metropolitana de la región educativa panameña
}

\author{
Ambulo Arosemena, Graciela; González Salina, Margarita; Montenegro, Raúl; Brooks, Yileika; Bonilla, \\ Margarita; Camarena, Melissa; Moreno, Yoselyn \\ Análisis del sobrepeso, obesidad, actividad física y autoestima de la niñez en el área metropolitana de la región \\ educativa panameña \\ MHSalud, vol. 17, núm. 2, 2020 \\ Universidad Nacional, Costa Rica \\ Disponible en: http://www.redalyc.org/articulo.oa?id=237062748001 \\ DOI: https://doi.org/10.15359/mhs.17-2.1
}

Esta obra está bajo una Licencia Creative Commons Atribución-NoComercial-SinDerivar 3.0 Internacional. 
MHSAlud, ISSN: 1659-097X, 17(2), Julio-Diciembre, 2020, PP 1-17 Ambulo Arosemena, González Salina, Montenegro, Brooks, Bonilla, Camarena, Moreno

\section{Análisis del sobrepeso, obesidad, actividad física y autoestima de la niñez en el área metropolitana de la región educativa panameña}

Analysis of Overweight, Obesity, Physical Activity and Self-Estimation of Children in the Metropolitan Area of the Educational Region of Panama City, Panama

Análise do sobrepeso, obesidade, atividade física e autoestima das crianças da área metropolitana da região

educacional panamenha

Graciela Ambulo Arosemena

Universidad Especializada de las Américas, Panamá

DOI: https://doi.org/10.15359/mhs.17-2.1

graamb@yahoo.com

Redalyc: http://www.redalyc.org/articulo.oa?

(D) http://orcid.org/0000-0002-9259-0022

Margarita González Salina

Universidad Especializada de las Américas, Panamá

margaritasgs@hotmail.com

(D) http://orcid.org/0000-0002-9872-7050

Raúl Montenegro

Universidad Especializada de las Américas, Panamá

nicodiang98@gmail.com

(iD http://orcid.org/0000-0002-4317-0232

Yileika Brooks

Universidad Especializada de las Américas, Panamá

yassiell18@gmail.com

(iD http://orcid.org/0000-0002-2968-9927

Margarita Bonilla

Universidad Especializada de las Américas, Panamá

pola90@live.com

(DD http://orcid.org/0000-0001-7542-6944

Melissa Camarena

Universidad Especializada de las Américas, Panamá

Melia181@outlook.com

(iD http://orcid.org/0000-0001-7914-2877

Yoselyn Moreno

Universidad Especializada de las Américas, Panamá

yoselynu2013@hotmail.com

(DD http://orcid.org/0000-0002-8722-5151

Recepción: 20 Septiembre 2019

Aprobación: 27 Noviembre 2019

\section{ReSUMEN:}

Los altos niveles de sobrepeso y obesidad que producen diabetes, hipertensión u otras enfermedades crónicas son un problema de salud pública, que inicia desde la infancia hasta alcanzar la edad adulta y alteran, directamente, la calidad de vida de la persona. El propósito de este estudio es determinar los niveles de sobrepeso y obesidad, los niveles de actividad física y de autoestima de 
escolares de 9 a 13 de edad, en 13 centros educativos de la ciudad de Panamá centro. Se evaluaron un total de 748 estudiantes. Las variables se midieron por medio por medio del tallímetro y la pesa para calcular el índice de masa corporal, el cuestionario PAQ-C para medir la actividad física y el LAWSEQ para medir la autoestima de los niños y las niñas. En la población estudiada, se mostró un $17 \%$ de estudiantes con sobrepeso, un $13 \%$ con obesidad; un $70.05 \%$ con actividad física moderada y un $25.67 \%$ con bajos niveles de actividad física. En cuanto a la autoestima, se evidenció un $50.5 \%$ con autoestima promedio y un $44 \%$ con autoestima baja. En conclusión, el análisis de los resultados indica una mayor prevalencia de obesidad en las niñas, y un mayor nivel de actividad física en los niños; en el caso de la autoestima, la edad juega un papel importante en la percepción personal, especialmente en el género femenino.

Palabras ClaVe: movimiento, autopercepción, obesidad, índice de Quetelet, desarrollo de la niñez.

\section{Abstract:}

The high levels of overweight and obesity that produce diabetes, hypertension, and other chronic diseases are a public health threat that starts from childhood through the adult age, altering a person's quality of life directly. The purpose of this study is to determine the levels of overweight and obesity, the levels of physical activity and self-esteem of children aging 9 to 13 years, in educational establishments from the center of Panama City, Panama. Thirteen (13 educational centers participated pertaining to eight $(8$ territories from the capital of the country, where 748 students were evaluated. The instruments used to measure these variables were the measuring rod, the weight scale to measure the body mass index, the PAQ-C survey to measure the physical activity, and LAWSEQ to measure self-esteem in children. The studied population showed $17 \%$ of the students to be overweight, $13 \%$ obese, $70.05 \%$ with moderate physical activity, and $25.67 \%$ with low levels of physical activity. As far as self-esteem is concerned, $50.5 \%$ showed average self-esteem and $44 \%$ low self-esteem. In conclusion, it is important to perform physical activities as prevention and attention actions to obesity, overweight, and improvement of self-esteem. The analysis of the results indicates a higher prevalence of obesity in girls, and greater levels of physical activity in boys, in the case of self-esteem, age plays an important role in personal perception, especially in the female sex.

KEYWORDS: movement, self-perception, obesity, Quetelet index, child development.

\section{RESUMo:}

Os altos níveis de sobrepeso e obesidade que produzem diabetes, hipertensão ou outras doenças crônicas são um problema de saúde pública que começa na infância e continua na idade adulta e altera diretamente a qualidade de vida de uma pessoa. O objetivo deste estudo foi determinar os níveis de sobrepeso e obesidade, os níveis de atividade física e autoestima de crianças entre 9 e 13 anos de idade, em 13 centros educativos no centro da cidade do Panamá. Foram avaliados 748 estudantes. As variáveis foram medidas por meio do medidor de altura e do peso para calcular o índice de massa corporal, o questionário PAQ-C para medir a atividade física e o LAWSEQ para medir a autoestima das criancas. De acordo a população estudada, $17 \%$ tinham excesso de peso, $13 \%$ eram obesos, 70,05\% moderadamente ativos fisicamente e $25,67 \%$ com baixos níveis de atividade física. Em termos de autoestima, $50,5 \%$ tinham autoestima média e $44 \%$ baixa autoestima. Em conclusão, a análise dos resultados indica uma maior prevalência de obesidade nas meninas e um maior nível de atividade física nos meninos; no caso da autoestima, a idade desempenha um papel importante na percepção pessoal, especialmente para o gênero feminino.

Palavras-CHAVE: movimento, auto percepção, obesidade, índice de Quetelet, desenvolvimento infantil.

\section{INTRODUCCIÓN}

La sociedad actual está amenazada de manera constante por diversos factores que ponen en riesgo la integridad de las personas; los problemas latentes en el área de la salud, como la diabetes, hipertensión y la obesidad, se han acrecentado a un nivel incapacitante. Preocupa esta situación, pues estos problemas pudieron haber sido prevenidos con la intervención en el momento adecuado (Chan, 2016. Está demostrado que muchas de las enseñanzas obtenidas en la niñez son las que marcan el futuro del individuo, en su personalidad, en su salud y en su desempeño social. He aquí la importancia de la enseñanza de hábitos saludables que formen al individuo con una actividad física efectiva y una alimentación adecuada, que prevengan enfermedades adquiridas y de carácter no transmisibles (ECNT (González-Jurado, 2013; Rodríguez y Casimiro, 2000.

Según Nestle (2007 y Leutwyler-Ozelli (2007, el aumento de calorías en los alimentos de elaboración rápida, productos precocidos y empaquetados, y los menús de restaurantes preparados en un ambiente 
cuyo ritmo laboral es acelerado, contribuyen al aumento de la prevalencia del sobrepeso y la obesidad en la población.

Tal y como lo señala la OMS (2016), 41 millones de menores de cinco años a nivel mundial presentaron sobrepeso u obesidad; información aún es más impactante por el incremento de los índices en los países en vía de desarrollo.

En el caso de Panamá, no hay un registro oficial que determine cuántas personas sufren de obesidad infantil, eso en parte se explica porque pacientes con obesidad no se hospitalizan, lo que dificulta tener un registro exacto. Sin embargo, De Caballero, Sinisterra, Lagruta y Atalah (2004), basados en su experiencia médica, mencionan que la tendencia de pacientes con esta enfermedad apunta a un aumento. Señalan que para ese año fueron atendidos en el hospital 450 consultas, de las cuales la mitad tenía que ver con problemas relacionados con la obesidad. En el año 2008, según Torrijos (2014), la obesidad fue la décima razón por la cual las personas acudieron a consulta externa en los distintos centros de atención, la tasa fue de 1 237,1 casos por cada 100 mil habitantes, correspondiente a la décima patología por la cual asistieron mayor volumen de pacientes a los hospitales públicos en Panamá (MINSA, 2008). Este informe también señaló que, en Panamá, 17350 personas murieron en 2012, de las cuales 8517 (49\%) fueron por enfermedades relacionadas con la obesidad. Estos datos reflejan que aproximadamente un $35 \%$ de la población se encuentra en sobrepeso y el $28 \%$ en estado de obesidad (Torrijos, 2014); en otras palabras: seis de cada diez panameños tienen problemas con el peso corporal.

Al mismo tiempo, la última Encuesta de Niveles de Vida, realizada por el Ministerio de Economía y Finanzas de Panamá [MEF] (2008), señaló que en el grupo de infantes en edades entre 10 a 17 años, el 18,2 $\%$ presentó sobrepeso y el 7,2 \% entró en la categoría de obesidad. Aunado a esto estudios de Sasson, Lee, Jan, Fontes y Motta (2014), el Instituto de Nutrición de Centroamérica y Panamá [INCAP] menciona que este país se encuentra en los primeros lugares de cifras de obesidad en menores entre las edades de 5 a 9 años, donde el $18 \%$ tiende a sufrir de sobrepeso, y un $9 \%$ de obesidad. Ello ha generado un aumento en las probabilidades de padecer dificultad respiratoria, fracturas, hipertensión arterial, enfermedades cardiovasculares, resistencia a la insulina y problemas psicológicos a temprana edad, además de aumentar la posibilidad de una muerte prematura y discapacidad en la edad adulta en la población panameña (Dávila-Torres, González-Izquierdo y Barrera-Cruz, 2015; OMS, 2014; Sánchez, 2015).

Rosa, García-Canto, Rodríguez, Pérez, Tárraga y Tárraga (2017) mencionan que escolares con un mejor nivel de actividad física tienen un mejor nivel de condición física; encontraron que las mujeres $(37,1 \%)$ tienen un alto porcentaje de condición física en comparación con los hombres (36,7 \%). También manifiestan la importancia de promover un estilo de vida más activo desde edades tempranas, en el que se impliquen ámbitos familiares, escolares, sanitarios y que, mediante esto, se pueda crear un medio favorable con el propósito de incrementar oportunidades para la realización de actividades físicas.

Rosa, García-Cantó y Carrillo (2018) plantean la importancia que tiene el realizar actividad física y tener una buena capacidad aeróbica como medida para tener una autoevaluación positiva de la salud, debido a que en hallazgos encontrados en sus investigaciones consideran la importancia de adoptar estilos de vida activos en poblaciones infantiles y preadolescentes. Estos estilos deben basarse en realizar, habitualmente, actividad física y mejorar, mediante esto, sus condiciones físicas. Al realizarlas en estos periodos se puede lograr mejoras en la construcción de las bases de la personalidad de estos grupos de infantes, pues adquieren estos hábitos de vida saludable más consecutivos en su vida.

Espinosa (2013), en su escrito "Educación física restringida”, señala que la enseñanza de esta materia en Panamá ha sido objeto de reducción de horas y en algunos bachilleratos ha dejado de ser obligatoria, para convertirse en optativa. Esto coloca en mayor riesgo el uso de la actividad física en los diferentes escenarios de formación y viola, de esta forma, las normas establecidas con base en la Constitución Política del país y la Ley Orgánica n.o 47 de 1946 y 1995. En la actualidad, solamente algunos colegios mantienen, por iniciativa del grupo docente, la actividad física como parte del aprendizaje de infantes, como algo extra del currículo 
reglamentado para el desarrollo de la niñez panameña, lo que demuestra la situación alarmante que vive el país y la poca atención que los centros educativos le brindan a los aspectos de estilos de vida activos, que son de suma importancia para el crecimiento y desarrollo de la niñez, y en todas las etapas de la vida para ambos sexos (Madrona, Jordán y Barreto, 2008. Dentro del Ministerio de Educación de Panamá [MEDUCA] (2017, se ha añadido la "Ley de Educación Alimentaria Nutritiva" en el sistema educativo; sin embargo, pese a que esto representa algunos pasos positivos hacia la población infantil, las autoridades de Panamá están conscientes de que no basta con reglamentar la venta de alimentos en las escuelas, sino que se requiere, además, una educación hacia la práctica de la actividad física y el ejercicio.

Aunado a esta situación, la obesidad en edad pediátrica es una de las enfermedades nutricionales de mayor prevalencia en el país; afecta aproximadamente a un 25-30 \% de la población infantil y adolescente (Mayo, 2017. Por tanto, cabe destacar la importancia de la actividad física para la contribución del gasto energético, para favorecer la pérdida de peso mediante la realización de actividades adecuadas a las diferentes edades, en conjunto con la dieta adecuada y controlada (Zamora y Laclé, 2012.

Por otro lado, niñas y niños obesos y con problemas de sedentarismo son los primeros en presentar cambios psicológicos, lo que les marca y les dificulta el cambio de hábitos (Sánchez y Ruíz, 2012). La baja autoestima es uno de los mayores detonantes a la predisposición de mantenerse en este círculo de sobrepeso y obesidad y conlleva muchas veces a problemas más graves como la depresión, ansiedad, problemas de conducta, trastornos de alimentación y discriminación social (Ortega, Muros, Palomares, Martín y Cepero, 2015. En este sentido, resulta relevante abordar dicha variable psicosocial, para determinar aquellos elementos afectivos, conductuales, cognitivos y sociales que impactan el desarrollo infantil, para poder controlar la influencia de la insatisfacción corporal en el momento de obtener resultados en esta población (Sánchez y Ruíz, 2012.

Con base en lo anterior, el artículo tiene, como objetivo, analizar el nivel de obesidad/sobrepeso, práctica de actividad física y autoestima presentes en menores de 9 a 13 años en el área metropolitana en Panamá.

\section{Hipótesis}

H1: Existe una relación entre la obesidad/sobrepeso, practica de actividad física y autoestima con respecto al género, edad y nivel escolar en niños y niñas de 9 a 13 años en el área metropolitana en Panamá

H0: No existe una relación entre la obesidad/sobrepeso, practica de actividad física y autoestima con respecto al género, edad y nivel escolar en niños y niñas de 9 a 13 años en el área metropolitana en Panamá.

\section{Marco metodológico}

\section{Tipo de investigación}

El estudio tiene un diseño de investigación descriptiva de una sola aplicación, que permite evaluar el sobrepeso y obesidad infantil, así como el nivel de actividad física y la autoestima de estudiantes de cuarto, quinto y sexto de primaria de 13 centros educativos de 8 corregimientos del área urbana de Panamá.

\section{Participantes}

En el grupo de estudio participaron 748 estudiantes de un salón de IV, V y VI grado primario de 13 centros educativos de la ciudad de Panamá, de los cuales 236 (31.6 \% cursaron cuarto grado, 305 (40.8 \% el quinto 
grado y 207 (27.7 \%) el sexto grado y con 397 (53.1\%) hombres y mujeres 351 (46.9\%) mujeres. Con una edad entre los 9 y 13 años, una talla promedio de $143.82 \pm 9.5$ y un peso corporal promedio de $40.66 \pm 10.92$.

El muestreo utilizado es de tipo probabilístico por conglomerados de varias etapas (Hernández-Sampieri, Fernández y Baptista, 2014), con estratificación por los 24 corregimientos del distrito de Panamá, además de un criterio de selección de proporcionalidad detallado en las unidades muestréales siguientes: Centro de estudio cercano a la universidad, un aula por nivel educativo: se tomó como referencia el conjunto de grupos de 4, 5, y 6 de primaria de cada centro, en áreas que no sean de zona roja de la provincia de Panamá.

\section{Instrumentos}

\section{Índice de masa corporal}

Para calcular el IMC de cada estudiante se midió la talla y el peso. La talla se midió, por medio estadiómetro marca SECA 213. Al niño o niña se le pidió que permaneciera de espalda contra el estadiómetro y seguidamente se registró el dato de la estatura en centímetros. El peso se midió a través de una balanza modelo Tanita UM-081, sin medias, ni calzado y se registró en el peso en kilogramos. Se utilizó la herramienta de cálculo del IMC para población infantil y juvenil ( 2 a 19 años) brindada por el Centro de Control y Prevención de Enfermedades (CDC) de los Estados Unidos en su sitio web, la cual estima el IMC por percentil de edad según los cuadros de crecimiento por edad de CDC, a partir de la fecha de nacimiento, el día de medición, el peso y la talla de la persona (Centro de Control y Prevención de Enfermedades, 2015).

Nivel de actividad física

Cuestionario de actividad física para niños y niñas mayores (PAQ-C)

El cuestionario PAQ-C (en inglés "Physical Activity Questionnaire for Older Children con siglas PAQC (Crocker, Bailey, Faulkner, Kowalski y McGrath, 1997) es un instrumento dirigido a niños de personas de edades de 8 a 14 años, basado en medidas de autorreporte, que proporciona puntuaciones generales de actividad física en los siete ultimo días, en diferentes momentos y situaciones del día. El PAQ-C demostró una confiabilidad de test-retest adecuada $(r=0.75$ a 0.82$)$ y una razonable validez de $(r=0.45$ a 0.53$)$ en comparación con otras medidas objetivas de actividad física (Crocker et al., 1997; Janz, Lutuchy, Wenthe y Levy, 2008). Este instrumento fue validado al español por Martínez-Gómez, et al (2009) para niños, niñas y adolescentes de origen español.

\section{Autoestima}

Cuestionario sobre autoestima (LAWSEQ) (con nombre en inglés Lawrence Self-Esteem Questionnaire), adaptación en español por Marcó y de Gracia (2000). Es un instrumento de valoración de autoestima en poblaciones infantiles, está basado en la sumatoria de características cognitivas y físicas de cada persona misma. El instrumento cuenta con 16 preguntas de valoración directa de la percepción de sí. Tiene una fiabilidad interna de $\alpha=0.76$ (Lawrence, 1981) y una validez externa aceptable en cuanto al Coppersmith Self-Esteem Inventory $(\mathrm{r}=0.73)$.

\section{Procedimiento}

Primeramente, se estructuró el protocolo científico que describió las directrices de la investigación, así como la selección de los instrumentos, los cuales están confiabilizados a nivel internacional. Una vez estructurada la fase inicial, se prosiguió a obtener los datos necesarios del Ministerio de Educación [MEDUCA], para la delimitación de la población y la selección del grupo estudio a participar, utilizando un tipo de muestreo por conglomerados con estratificación, por corregimientos en la ciudad de Panamá; además de un criterio de selección de los colegios cercanos a la Universidad. Una vez elegidos se utilizó un muestreo aleatorio simple para la elección de estudiantes de los niveles educativos, de cuarto, quinto y sexto de primaria, de acuerdo 
con la cantidad de salones por nivel educativo de cada centro participante. Una vez tramitados los permisos correspondientes, se procedió a realizar la evaluación diagnóstica, la cual consistió en la medición de la talla y el peso de cada estudiante y las evaluaciones de autorreporte del nivel de actividad física y autoestima. Todas las mediciones fueron realizadas en un día normal de clases y con presencia profesional.

\section{Análisis de datos estadísticos}

El análisis estadístico de los resultados se realizó a través de la estadística descriptiva, se obtuvieron y analizaron las medidas de tendencia central para poder darle respuesta a la pregunta y objetivos de la investigación. Por otro lado, se realizó una correlación y comparación entre las variables índice de masa corporal, nivel de actividad física y la autoestima, según el análisis con las variables independientes de sexo, edad y grado escolar, para ver si el exceso de peso o el bajo nivel de actividad física influye en el autoestima o juicio de valor que tiene cada estudiante sobre sí. Se utilizó el programa estadístico SPSS, versión 20 para Windows, para tabular los datos y poder realizar el análisis de resultados. El nivel de significación estadística se estableció en $\mathrm{p}<0.05$ con un intervalo de confianza del $95 \%$.

\section{Resultados}

Los resultados que se presentan a continuación muestran el análisis descriptivo de las variables de la investigación, en el cual se reflejan los principales resultados de la población estudiada:

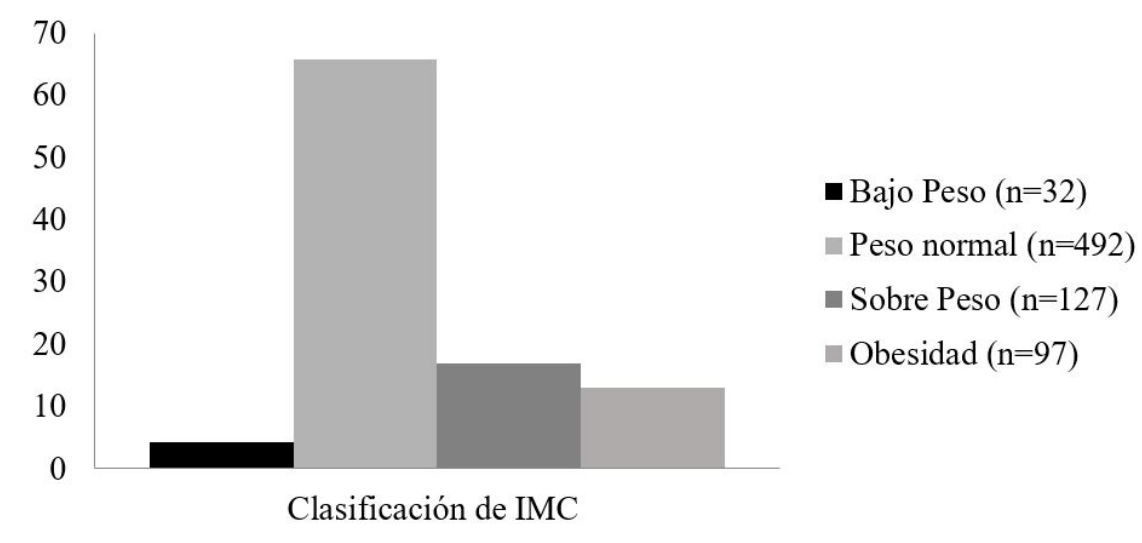

FIGURA 1

Clasificación del indice de masa corporal de la población evaluada.

En la Figura 1 se muestra un 65.80 \% de la población estudiada con un peso normal, mientras que un $17 \%$ presentó sobrepeso y un $13 \%$ obesidad, lo que indica que un $30 \%$ presentó problemas de peso, en las cuales se deberá trabajar. 
MHSALUD, ISSN: 1659-097X, 17(2), JULio-DiciemBre, 2020, pP 1-17 ambulo Arosemena, González Salina, Montenegro, Brooks, Bonilla, Camarena, Moreno

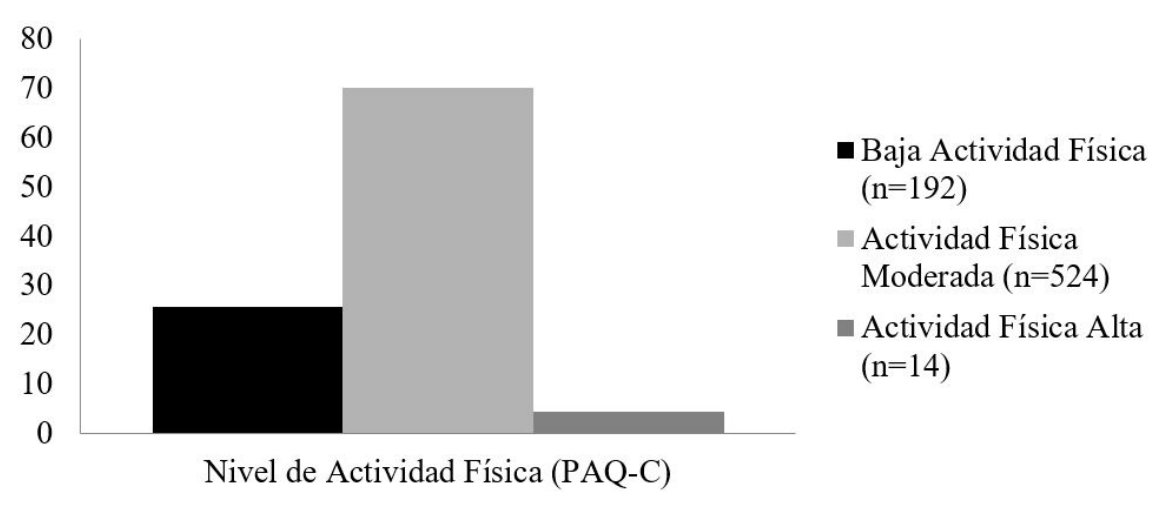

FIGURA 2

Nivel de actividad física de la población evaluada.

En la Figura 2 se encuentra que un $70.05 \%$ de la población percibe tener una actividad física moderada, mientras que el $25.67 \%$ de la población se encuentra en un nivel de actividad física bajo y solamente un 4.28 $\%$ indica tener un nivel de actividad física alto.

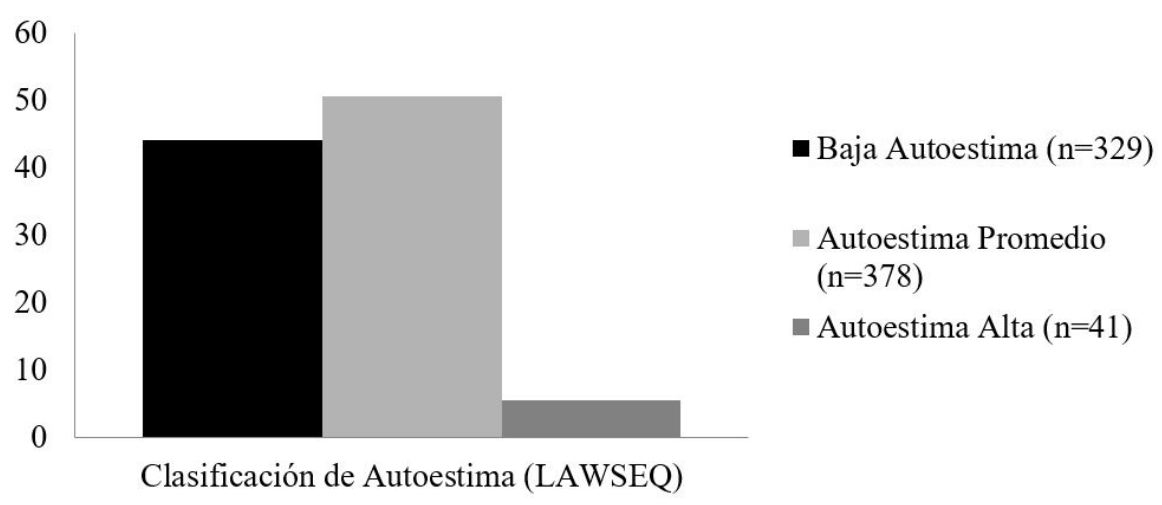

FIGURA 3.

Clasificación de autoestima de la población evaluada.

En la Figura 3 se muestra un $50.5 \%$ de la población con una autoestima promedio, mientras que un $44 \%$ mostró un nivel de autoestima bajo y únicamente el 5,5\% con una autoestima alta. 
MHSAlud, ISSN: 1659-097X, 17(2), Julio-Diciembre, 2020, PP 1-17

ambulo Arosemena, González Salina, Montenegro, Brooks, Bonilla, Camarena, Moreno

TABLA 1

Comparación del indice de masa corporal, el nivel de actividad fisica y autoestima según sexo y edad

\begin{tabular}{|c|c|c|c|c|c|}
\hline & 9 & 38 & $17,54 \pm 2,94$ & $3,04 \pm 0,864$ & $15,13 \pm 5,40$ \\
\hline & 10 & 128 & $19,38 \pm 4,26$ & $3,12 \pm 1,006$ & $15,56 \pm 4,67$ \\
\hline \multirow[t]{5}{*}{ Niñas } & 11 & 132 & $19,82 \pm 3,74$ & $2,92 \pm 0,805$ & $15,72 \pm 4,96$ \\
\hline & 12 & 47 & $20,58 \pm 4,13$ & $2,82 \pm 0,858$ & $16,89 \pm 4,95$ \\
\hline & 13 & 6 & $21,67 \pm 3,71$ & $2,84 \pm 0,892$ & $15,33 \pm 4,50$ \\
\hline & 9 & 35 & $18,46 \pm 3,82$ & $3,01 \pm 0,806$ & $13,57 \pm 4,47$ \\
\hline & 10 & 121 & $19,12 \pm 4,29$ & $3,23 \pm 0,775$ & $15,38 \pm 4,63$ \\
\hline \multirow[t]{3}{*}{ Niños } & 11 & 128 & $19,01 \pm 3,49$ & $3,26 \pm 0,767$ & $16,16 \pm 4,36$ \\
\hline & 12 & 100 & $20,22 \pm 3,82$ & $3,12 \pm 0,755$ & $16,96 \pm 4,34$ \\
\hline & 13 & 13 & $19,59 \pm 2,78$ & $3,05 \pm 1,011$ & $16,38 \pm 5,04$ \\
\hline$p$ & & & 0,001 & 0,360 & 0,002 \\
\hline
\end{tabular}

En la Tabla 1 se pueden encontrar diferencias significativas en la variable de $\operatorname{IMC}(\mathrm{F}(2)=3.920 ; p=0,001)$ en relación con la edad. Las niñas de 13 años muestran un mayor índice de masa corporal en comparación con los demás niños, en el nivel de actividad física los niños de 11 años muestran una mayor actividad física en comparación con los demás niños; en el nivel de autoestima $(\mathrm{F}(2)=4.722 ; p=0,002)$ los niños de 9 años muestran una baja autoestima en comparación con los niños de 12 años que muestran una elevada autoestima en comparación con ellos. En el caso de las niñas se observa una tendencia a incrementar su nivel de autoestima conforme avanzan en la edad; sin embargo, al acercarse a la etapa de la adolescencia, se observa un descenso en esta misma. 
MHSALUD, ISSN: 1659-097X, 17(2), JuLio-DiciemBRe, 2020, PP 1-17 ambulo Arosemena, González Salina, Montenegro, Brooks, Bonilla, Camarena, Moreno

TABLA 2

Comparación del indice de masa corporal, el nivel de actividad fisica y autoestima según grado escolar

\begin{tabular}{ccccc}
\hline Grado escolar & $\mathbf{n}$ & $\begin{array}{c}\text { Índice de masa } \\
\text { corporal }\end{array}$ & PAQ-C & Autoestima \\
\hline 4to Grado & 236 & $18,43 \pm 3,63$ & $3,19 \pm 0,896$ & $14,72 \pm 4,53$ \\
5to Grado & 305 & $19,57 \pm 3,99$ & $3,07 \pm 0,788$ & $16,26 \pm 4,71$ \\
6to Grado & 207 & $20,34 \pm 3,89$ & $3,03 \pm 0,854$ & $16,45 \pm 4,75$ \\
$\boldsymbol{p}$ & & $\mathbf{0 , 0 0 0 1}$ & $\mathbf{0 , 1 0 8}$ & $\mathbf{0 , 0 0 0 1}$ \\
\hline
\end{tabular}

En la Tabla 2 se muestran diferencias significativas en la variable de índice de masa corporal según el grado escolar $(\mathrm{F}(2)=13,815 ; p=0,0001)$, en relación con el grado escolar, también se muestra que los niños de $6^{\text {to }}$ grado tienen un mayor índice de masa corporal en comparación con los de $4^{\text {to }}$ grado; en cuanto a la actividad física se muestra que los niños de $4^{\text {to }}$ grado muestran una mayor actividad física en comparación con los de sexto, respecto al nivel de autoestima se observa que, los niños de 4to grado muestran una baja autoestima en comparación con los niños de $6^{\text {to }}$ grado $(\mathrm{F}(2)=9,778 ; p=0,0001)$. 
MHSAlud, ISSN: 1659-097X, 17(2), Julio-Diciembre, 2020, PP 1-17

ambulo Arosemena, González Salina, Montenegro, Brooks, Bonilla, Camarena, Moreno

TABLA 3

Correlación del indice de masa corporal, el nivel de actividad física y autoestima según grado escolar, edad y género

\begin{tabular}{|c|c|c|c|c|c|c|c|}
\hline & & SEXO & $E D A D$ & GRADO & IMC & $P A Q \_C$ & $\begin{array}{c}\text { AUTOES } \\
\text { TIMA }\end{array}$ \\
\hline \multirow[t]{3}{*}{$I M C_{-}$} & $\begin{array}{l}\text { Correlación } \\
\text { de Pearson }\end{array}$ &,- 029 &, $147(* *)$ &, $188(* *)$ & 1 &,- 015 &, 010 \\
\hline & $\begin{array}{l}\text { Sig. } \\
\text { (bilateral) }\end{array}$ & ,432 &, 000 &, 000 & & ,686 & ,776 \\
\hline & $N$ & 748 & 748 & 748 & 748 & 748 & 748 \\
\hline \multirow[t]{3}{*}{$P A Q \_C$} & $\begin{array}{l}\text { Correlación } \\
\text { de Pearson }\end{array}$ &, $115(* *)$ &,- 041 &,$- 074\left(^{*}\right)$ &,- 015 & 1 &, 033 \\
\hline & $\begin{array}{l}\text { Sig. } \\
\text { (bilateral) }\end{array}$ &, 002 &, 257 &, 043 & ,686 & &, 362 \\
\hline & $N$ & 748 & 748 & 748 & 748 & 748 & 748 \\
\hline \multirow[t]{3}{*}{ AUTOESTIMA } & $\begin{array}{l}\text { Correlación } \\
\text { de Pearson }\end{array}$ &, 016 &, $138(* *)$ &, $144(* *)$ & 010 & ,033 & 1 \\
\hline & $\begin{array}{l}\text { Sig. } \\
\text { (bilateral) }\end{array}$ &, 660 &, 000 &, 000 & ,776 & ,362 & \\
\hline & $N$ & 748 & 748 & 748 & 748 & 748 & 748 \\
\hline
\end{tabular}

Nota: ${ }^{* *}$ La correlación es significativa al nivel 0,01 (bilateral). ${ }^{*}$ La correlación es significante al nivel 0,05 (bilateral).

En la Tabla 3, se muestran que existe una correlación positiva significativa ( $\mathrm{r}: 0.147, p: 0.000)$ muy baja, entre los componentes de edad e índice de masa corporal; de igual forma se encontró una correlación positiva significativa $(\mathrm{r}=0,188, p=0.000)$ muy baja; del mismo modo, los componentes de sexo y nivel de actividad física muestran una correlación positiva significativa $(r=0,115, p=0.002)$ muy baja; en los componentes de grado y actividad física $(\mathrm{r}=-0.074, p=0.043)$ se encontró una correlación negativa muy baja, y en los componentes de autoestima y edad se evidenció que existe una correlación positiva significativa muy baja $(\mathrm{r}=0.138 p=0.000) \mathrm{y}$, de la misma manera, entre los componentes de autoestima y grado escolar existe una correlación positiva significativa muy baja $(\mathrm{r}=0.144, p=0.000)$, lo cual expresa que según aumentan de edad, más aumentan su índice de masa corporal. También, en cuanto al grado y actividad física, estos datos plantean que aumentar de nivel escolar influye en la disminución de la actividad física, por lo cual se cumple la H1: Existe una relación entre la obesidad/sobrepeso, practica de actividad física y autoestima con respecto al género, edad y nivel escolar en población infantil de 9 a 13 años en el área metropolitana en Panamá, ya que se encontraron correlaciones entre estos componentes. 
MHSALUD, ISSN: 1659-097X, 17(2), JuLIo-Diciembre, 2020, PP 1-17 Ambulo Arosemena, González Salina, Montenegro, Brooks, Bonilla, Camarena, Moreno

\section{Discusión}

El propósito de esta investigación fue analizar el sobrepeso, la obesidad, el nivel de actividad física y de autoestima en la población infantil de 9 a 13 años de cuarto, quinto y sexto grado de 13 centros educativos del área metropolitana de la Ciudad de Panamá. Con respecto a los hallazgos de esta investigación, se encontró una prevalencia de sobrepeso y obesidad del $30 \%$, lo que expone a la población evaluada a un mayor riesgo de sufrir enfermedades crónicas no transmisibles [ECNT], tales como padecimientos cardiacos, accidentes cerebrovasculares, hipertensión arterial, diabetes mellitus, y a un futuro de riesgo en la adultez de aumentar la morbilidad y mortalidad, como lo señalan la OMS (2016); Rodríguez y Casimiro (2000) y Spear, et al. (2007). Según Contreras, Orozco, Santamaría, Iglesias y Tolentino (2010), la obesidad es considerada un factor de riesgo que predispone a patologías cardiovasculares, diabetes, y algunos tipos de cáncer.

Mayo (2017) menciona que estadísticas en salud de Panamá, reportadas por el MINSA en los años 2008, 2015 y 2017, muestran una tendencia al incremento del sobrepeso y la obesidad con la edad, pasando de un 25 $\%$ a un $30 \%$ de prevalencia en la población infantil, lo que la ubica como la décima patología del país por la cual acudieron a consulta en esos años, con una tasa de 1237.1 casos por cada 100 mil habitantes (Torrijos, 2014). Asimismo, otros datos reportados por Torrijos (2014) y MINSA (2015a y b) revelan que aproximadamente el $63 \%$ de la población panameña general se encuentra en sobrepeso u obesidad; es decir, seis de cada diez personas panameñas tienen problemas con el peso corporal, lo que también evidencia el incremento de esta problemática, ya que a diferencia de los datos revelados en el Estado Nutricional de la Población del MINSA (2014), un $60 \%$ de la población adulta del país presentaba estas condiciones perjudiciales para la salud.

Comparar el resultado de prevalencia de sobrepeso y obesidad de niñas y niños panameños, con otros estudios similares realizados en países centroamericanos, permite mencionar que Guatemala presenta un índice del 40 \%, Costa Rica un $34.2 \%$, Nicaragua un 25.9 \% El Salvador un $23.3 \%$, lo que indica una problemática de salud infantil generalizada en toda la región (Dubón y Aguilar, 2018; Evert y Álvarez, 2018; Herrera-Monge, Álvarez, Sánchez, Herrera-González, Villalobos y Tenorio, 2019; Reyes, Otero, Pastrán, Herrera-Monge y Álvarez, 2018). Según Serra-Valdés (2015), la obesidad representa un problema muy importante durante la niñez en todo Centroamérica, ha alcanzado a 289 millones de esta población en edades entre los 5 a 12 años al terminar el 2015, lo que ha generado un aumento en las tasas de estas patologías en los últimos 30 años, superando a países como Estados Unidos y México.

Con relación con el género, esta investigación arrojo diferencias en los datos del IMC, las niñas obtuvieron un índice superior en comparación con los niños de la misma edad. Estos resultados se pueden explicar por los cambios metabólicos y en los ambientes físicos y sociales, que acentúan así las diferencias en ambos géneros (Cofré, 2015; Pinel, et al., 2017).

A pesar de los beneficios de la actividad física durante la niñez, este estudio ha demostrado que un $25 \%$ del estudiantado evaluado presentó niveles de actividad física baja. Contrario a esta investigación, INCAP (2016) reporta que al menos un $70 \%$ de la población escolar centroamericana es sedentaria: los niveles de sedentarismo en la niñez panameña (43.5 \%) son menores si se comparan con países como El Salvador (Evert y Álvarez, 2018) que presentó un 53.7 \% y Tegucigalpa-Honduras (Lacayo, Check, Vega y Córdova, 2018) con un $47.0 \%$; pero no deja de ser preocupante. Si se contrastan, los porcentajes obtenidos con las recomendaciones de actividad física de la OMS (2010) para los niños, niñas y jóvenes, que recomiendan practicar actividades al menos 60 minutos semanales de moderada a vigorosa intensidad, los resultados nos indican que el nivel de sedentarismo en la región está en aumento y se requiere, con premura, implementar alguna intervención, para palear la problemática de desarrollar ECNT a temprana edad, lo cual se asoci también a la alta prevalencia de sobrepeso y obesidad en infantes.

En cuanto a las diferencias entre niños y niñas, la bibliografía ha reportado que los niños en general son más activos que las niñas, diferencias que se incrementas, entre géneros, con la edad (De Piero, Rodríguez, 
González y López, 2014) y que pueden ser explicadas por los procesos de sociabilización que determinan el rol de cómo los niños y niñas perciben la actividad física.

Con respecto a la variable de autoestima, los resultados encontrados en el presente estudio muestran un 43.9 \% de autoestima baja en la niñez evaluada. Pese a que en Panamá no existen estudios de la autoestima en la niñez, se ha reportado, en grupos similares, un nivel de baja autoestima en un $35 \%$ de escolares, de acuerdo con el estudio de Herrera-González, Morera, Araya, Fonseca y Vargas (2017). Adicionalmente, en estudios realizados en el 2018 en la región Centroamericana se han reportado niveles más bajos de $52.9 \%$ en el contexto escolar salvadoreño, (Evert y Álvarez, 2018), por lo que, al relacionar los resultados altos de sobrepeso u obesidad y los bajos niveles de actividad física encontrados en este estudio, se podría indicar una relación con la percepción baja de la autoeficacia y la competencia física con el resultado de un impacto negativo en la autoestima de los sujetos evaluados (Contreras-Valdez, Hernández-Guzmán y Freyre, 2016).

Según Meza-Peña y Pompa-Guajardo (2016), la proporción entre autoestima y sexo marca una relación directa con el sobrepeso y la obesidad, al igual que con el autoconcepto físico, pues existen diferencias significativas entre niños y niñas en cuanto a lo social, emocional y académico, como se observa en el estudio realizado por dicho equipo. No obstante, la práctica de actividad física individual o colectiva podría ser una herramienta beneficiosa para aumentar los niveles de autoestima en la niñez y en la adolescencia, como lo mencionan Batista, et al. (2016).

Rosa, García-Cantó y Carrillo (2018) explican la importancia de realizar actividad física desde edades , pues así se pueden establecer los pilares de una vida saludable mediante la construcción de las bases de la personalidad del ser humano en edades tempranas. De igual manera, en esta investigación, al encontrar unas relaciones significativas entre la autoestima con la edad y el grado escolar, se manifiesta la importancia de que se implementen más programas de actividad física como medida para disminuir el sobrepeso y obesidad en estudiantes, para lograr, así, trabajar más su autoestima.

A la luz de los resultados obtenidos, los niveles altos de sobrepeso y obesidad correlacionados con niveles bajos y preocupantes de actividad física podrían estar relacionados con la disminución en la percepción del autoconcepto y la autoeficacia de los grupos escolares panameños (Cofré, 2015). Esto nos demuestra la necesidad que existe en esta población de una educación en salud para la niñez, para así alcanzar estilos de vida saludable (González-Jurado, 2013). He aquí la importancia de hacer estudios que nos permitan justificar la forma adecuada del comportamiento, desde el nacimiento hasta el transcurrir de la vida; ello da, de esta forma, la relevancia a la presente investigación.

\section{Conclusiones}

Se concluye que la mayor parte de la población estudiada estaba en un peso normal y mantenía una actividad física en un rango moderado, pero se encontró que la autoestima estaba en rangos de bajo promedio, lo que permite resaltar la importancia de una actividad física que busque mejorar la estabilidad emocional estudiantil, ya que, por lo general, se tiende a relacionar los problemas de autoestima con los problemas de sobrepeso u obesidad y los niveles de actividad física. Ello indica que, si se trabaja más en actividades físicas, esto podría ayudar significativamente a bajar los niveles de sobrepeso y obesidad y estabilizar los problemas de autoestima baja para lograr una autoestima más sana en el estudiantado.

Además, al analizar detalladamente la población, existe una mayor prevalencia de obesidad en las niñas, y al ver el nivel de actividad física se muestra que en los niños existe un mayor estilo de vida activo en comparación con las niñas. Los niños de 9 años se muestran con una autoestima más baja en comparación que los niños de 13 años, mientras que las niñas de 9 años se muestran con mayor autoestima, en comparación de las niñas de 13 años que revelan una autoestima baja.

También se recalca la importancia que tiene la edad, el género y el nivel escolar en el que se encuentra el estudiantado con la actividad física, la autoestima y el índice de masa corporal (IMC), ya que se muestra 
MHSALUd, ISSN: 1659-097X, 17(2), Julio-Diciembre, 2020, PP 1-17 Ambulo Arosemena, González Salina, Montenegro, Brooks, Bonilla, Camarena, Moreno

la importancia que se le debe dar a la actividad física en todos los niveles, para disminuir los problemas relacionados con la obesidad/sobrepeso y con los problemas de autoestima presentados en estudiantes.

\section{RECOMENDACIONES}

Desde un punto de vista de salud y en el contexto de la universidad pública, se recomienda iniciar un proceso de difusión de los hallazgos de esta investigación, con el fin de poder unir esfuerzos que conlleven a crear un sistema de vigilancia epidemiológica a nivel de país y, esencialmente, el dar a conocer los resultados en los diferentes entornos panameños, con el fin de unir esfuerzos en el desarrollo de programas, modelos o proyectos que contribuyan a palear la situación en el entorno escolar y que utilicen el movimiento humano como herramienta guía. Se requiere, para su logro, el desarrollo de políticas públicas con apoyo de los demás entes gubernamentales. Asimismo, es necesario identificar las dificultades que conlleva hacer evaluaciones en el área rural, para contrarrestarlas y dar apertura a las investigaciones que permitan evidenciar la situación en temas de una alimentación y actividad física adecuada que vive este sector actualmente. Así se podría evitar el desarrollo de estas afectaciones que entorpecen el proceso de crecimiento de la población infantil del país.

\section{REFERENCIAS}

Batista, M., Cubo, S., Honorio, S. y Martins, J. (2016). The practice of physical activity related to self-esteem and academical performance in students of basic education. Journal of Human Sport and Exercise, 11(2), 297-310. Recuperado de http://www.redalyc.org/pdf/3010/301050350002.pdf

Centro de Control y Prevención de Enfermedades [CDC]. (2015). Calculadora del indice de masa corporal para la población infantil y juvenil. Recuperado de https://nccd.cdc.gov/dnpabmi/Calculator.aspx

Cofré, C. (2015). El problema del sobrepeso y la obesidad infantil y sus interacciones con la actividad física, el sedentarismo y los hábitos nutricionales en Chile Tesis de la Universidad de Granada). Recuperado de https://doi.org/10.406 7/S0034-98872007000100009

Contreras, V., Orozco, M., Santamaría, S., Iglesias, A. y Tolentino, A. (2010). Salud y obesidad en adolescentes. Revista Cientifica Electrónica de Psicología, 10, 9-17. Recuperado de https://www.uaeh.edu.mx/investigacion/icsa/LI_ PrevAten/Vero_Rodri/4.pdf

Contreras-Valdez, J. A., Hernández-Guzmán, L. y Freyre, M. Á. (2016). Insatisfacción corporal, autoestima y depresión en niñas con obesidad. Revista Mexicana de Trastornos Alimentarios, 7(1), 24-31. Recuperado de ht tp://www.redalyc.org/pdf/4257/425746132004.pdf

Crocker, P.R. E., Bailey, D.A., Faulkner, R.A., Kowalski, K.C. y McGrath, R. (1997). Measuring general levels of physical activity: Preliminary evidence for the Physical Activity Questionnaire for Older Children. Medicine and Science in Sports and Exercise, 29(10), 1344-1349. https://doi.org/10.1097/00005768-199710000-00011

Chan, M. (2016). Obesidady diabetes, una plaga lenta pero devastadora. Washington D.C., EE.UU. Recuperado de h ttp://www.who.int/dg/speeches/2016/obesity-diabetes-disaster/es/

Dávila-Torres, J., González-Izquierdo, J. y Barrera-Cruz, A. (2015). Panorama de la obesidad en México. Revista Médica del Instituto Mexicano del Seguro Social, 53(2), 240-249. Recuperado de http://revistamedica.imss.gob .mx/editorial/index.php/revista_medica/article/viewFile/21/54

De Piero, A., Rodríguez, E., González, L. G. y López-, A. M. (2014). Sobrepeso y obesidad en un grupo de escolares españoles. Revista Chilena de Nutrición, 41(3), 264-271. https://doi.org/10.4067/S0717-7518201400030000 6

De Caballero, E., Sinisterra, O., Lagrutta, F., Atalah, E. (2004). Evaluación del impacto de alimentación complementaria de Panamá en niños menores de 5 años. Arch Latinoam Nutr, 54, 66-71.

Dubón, S. y Aguilar, C. (2018). Análisis de sobrepeso y obesidad, niveles de actividad física y autoestima en la niñez guatemalteca. Manuscrito enviado a publicación. 
MHSAlud, ISSN: 1659-097X, 17(2), Julio-Diciembre, 2020, PP 1-17

ambulo Arosemena, González Salina, Montenegro, Brooks, Bonilla, Camarena, Moreno

Espinosa, E. (2013). Educación fisica restringida: Una asignatura en extinción. Recuperado de https://www.prensa.co $\mathrm{m} /$ reinaldo_weeks/Educacion-fisica-restringida_0_3613138736.html

Evert, B. y Álvarez, C. (2018). Análisis del sobrepeso y obesidad, niveles de actividad fisica y autoestima de la niñez salvadoreña. Manuscrito en preparación.

González-Jurado, J. (2013). Educar para una vida activa desde la educación física: El ejercicio físico como hábito saludable. Boletim Sociedade Portuguesa de Educação Física, 37, 131-136. Recuperado de https://boletim.spef.p t/index.php/spef/article/view/255/242

Hernández-Sampieri, R., Fernández, C. y Baptista, P. (2014). Metodología de la investigación. (Sexta Edición). México, D.F.: Editorial Mc Graw Hill.

Herrera-González, E., Morera, M., Araya, G., Fonseca, H. y Vargas, M. (2017). Análisis del estado físico y psicológico de la población escolar del cantón central de Heredia, Costa Rica. Revista MHSalud, 13(2), 1659-97. https:/ /doi.org/10.15359/mhs.13-2.7

Herrera-Monge, M.F., Álvarez Bogantes, C., Sánchez Ureña, B., Herrera-González, E., Villalobos Víquez, G. y Vargas Tenorio, J. (2019). Análisis de sobrepeso y obesidad, niveles de actividad física y autoestima en la niñez del II ciclo escolar del cantón central de Heredia, Costa Rica. Población y Salud en Mesoamérica, 17(1). doi: https:// doi.org/10.15517/psm.v17i1.35323

Instituto de Nutrición de Centroamérica y Panamá [INCAP]. (2016). Situación de la obesidad en Centroamérica y República Dominicana. Revista A.C. Nota Técnica, 1-7. Recuperado de http://www.incap.int/sisvan/index.php/es/cooperacion-tecnica-en-la-region/documentos-especializados/d oc_view/279-nota-tecnica-situacion-de-la-obesidad-en-centro-america-y-republica-dominicana-2016

Janz, K.F., Lutuchy, E.M., Wenthe, P. y Levy, S.M. (2008). Measuring activity in children and adolescents using selfreport: PAQ-C and PAQ-A. Medicine and Science in Sports and Exercise, 40(4), 767-72. https://doi.org/10.12 49/MSS.0b013e3181620ed1

Lacayo, M., Check, E., Vega, N. F. y Córdova, A. (2018). Análisis de sobrepeso y obesidad, niveles de actividad fisica y autoestima en la niñez de Tegucigalpa, Honduras. Manuscrito en preparación.

Lawrence, D. (1981). The delevolpment of a self-esteem questionnaire. Brithish Journal Educational Psychology, 51(2), 245-251. https://doi.org/10.1111/j.2044-19 8279. 1981.tb02481.x20

Leutwyler-Ozelli, K., (2007). El cerebro y la comida. Investigación y ciencia, 374, 42-43. Recuperado de https://www.i nvestigacionyciencia.es/revistas/investigacion-y-ciencia/saciedad-y-hambruna-451/el-cerebro-y-la-comida-274

Madrona, P. G., Jordán, O. R. C. y Barreto, I. G. (2008). Habilidades motrices en la infancia y su desarrollo desde una educación física animada. Revista Iberoamericana de Educación, 47, 71-96. Recuperado de https://rieoei.org/h istorico/documentos/rie47a04.pdf.

Marcó, M y de Gracia, M. (2000). Evaluación de la imagen corporal en preadolescentes. Girona, España: Universitat de Girona.

Martínez-Gómez, D., Martínez-de-Haro, V., Pozo, T., Welk, G. J., Villagra, A., Calle, M. E., Marcos, A. y Veiga, O. L. (2009). Fiabilidad y validez del cuestionario de actividad física PAQ-A en adolescentes españoles. Revista Española de Salud Pública, 83(3), 427-439.http://dx.doi.org/10.1590/S1135-57272009000300008

Mayo, M.A. (2017). Artículo de opinión: Obesidad infantil. Ministerio de Salud de Panamá. Recuperado de http://w ww.minsa.gob.pa/noticia/articulo-de-opinion-obesidad-infantil

Meza-Peña, C. y Pompa-Guajardo, E. (2016). Género, obesidad y autoconcepto en una muestra de adolescentes de México. RICYDE: Revista Internacional de Ciencias Del Deporte, 12(44), 137-148. https://doi.org/http://dx. doi.org/10.5232/ricyde2016.04404

Ministerio de Economía y Finanzas de Panamá [MEF]. (2008). Panamá - Encuesta de Niveles de Vida 2008. Recuperado de http://microdata.worldbank.org/index.php/catalog/70

Ministerio de Educación de Panamá. (2017). Merienda escolar: Alimentación escolar, extensión e información en nutrición. Recuperado de https://plataformacelac.org/programa/330 
MHSALUD, ISSN: 1659-097X, 17(2), JuLIo-Diciembre, 2020, PP 1-17 Ambulo Arosemena, González Salina, Montenegro, Brooks, Bonilla, Camarena, Moreno

Ministerio de Salud de Panamá [MINSA]. (2008). Estadísticas de salud 2007. Dirección Nacional de Políticas del Sector Salud. Recuperado de http://www.minsa.gob.pa/sites/default/files/publicacion-general/anuario_estadi stico_2007.pdf

Ministerio de Salud de Panamá [MINSA]. (2014). Indicadores de salud básicos, Panamá. Recuperado de http://www .minsa.gob.pa/sites/default/files/publicacion-general/ind._basicos_pma_2014.pdf

Ministerio de Salud de Panamá [MINSA]. (2015a). Análisis de la situación de salud Panamá 2015. Recuperado de ht tps://www.minsa.gob.pa/sites/default/files/publicaciones/asis_2015.pdf

Ministerio de Salud de Panamá [MINSA]. (2015b). El estudio de la dieta total de Panamá 2013. Recuperado de https://www.paho.org/pan/index.php?option $=$ com_docman\&view=download\&category_slug=publicatio ns\&alias=377-el-estudio-de-dieta-total-de-panama-2013-edtpan-2013\&Itemid=224

Nestlé, M. (2007). Dietética elemental. Investigación y ciencia, 374, 20-29. Recuperado de https://www.investigacion yciencia.es/revistas/investigacion-y-ciencia/saciedad-y-hambruna-451/diettica-elemental-262

Organización Mundial de la Salud [OMS]. (2010). Informe sobre la salud en el mundo. Recuperado de https://apps.who.int/iris/bitstream/handle/10665/44373/9789243564029_spa.pdf;jsessionid=2B1CC0 C9B3BE2C4713666242DE6D539B? sequence $=1$

Organización Mundial de la Salud [OMS]. (2014). Informe sobre la situación mundial de las enfermedades no transmisibles. Who, 1-18. https://doi.org/978 9241564229

Organización Mundial de la Salud [OMS]. (2016). Aumento del sobrepeso y la obesidad infantiles. Recuperado de htt p://www.who.int/dietphysicalactivity/childhood/es/

Ortega, M. A., Muros, J.J., Palomares, J., Martín, J. A. y Cepero, M. (2015). Influence of BMI on self-esteem of children aged 12-14 years. Anales de Pediatría (English Edition) 83(5), 311-3-17. https://doi.org/10.1016/j.anpede.20 14.11 .003

Pinel, C., Chacón, R., Castro, M., Espejo, T., Zurita, F. y Pérez, A. (2017). Diferencias de género en relación con el Índice de Masa Corporal, calidad de la dieta y actividades sedentarias en niños de 10 a 12 años. Retos, 31, 179-180.

Reyes, R., Otero, E., Pastran, R., Herrera-Monge, M.F. y Alvarez-Bogantes, C. (2018). Análisis del sobrepeso, obesidad, niveles de actividad física y autoestima de la niñez de León, Nicaragua. Manuscrito enviado a publicación.

Rodríguez, P. L. y Casimiro, A. J. (2000). Papel del especialista en actividades físicas como promotor de salud y calidad de vida. En C. Águila, A.J. Casimiro y A. Sicilia (Coords.), Salidas profesionales y promoción en el ámbito de la actividad fisica y el deporte (pp. 211-242). Universidad de Almería: Servicio de publicaciones.

Rosa, A., García-Cantó, E., Carrillo, P. J. (2018). Percepción de salud, actividad física y condición física en escolares. Cuadernos de Psicología del Deporte, 18(3), 179-189.

Rosa, A., García-Canto, E., Rodríguez, P., Pérez, J., Tárraga, M. y Tárraga, P. (2017). Actividad física, condición física y calidad de la dieta en escolares de 8 a 12 años. Revista Nutrición Hospitalaria, 34(6),1292-1298. http://scielo .iscii.es/scielo.php?pip $=$ s0212-161120170

Sánchez, J. J. y Ruiz, A. (2012). Relación entre autoestima e imagen corporal en niños con obesidad. Revista Mexicana de Trastornos Alimentarios, 6(1), 38-44. ISSN 2007-1523. http://dx.doi.org/10.1016/j.rmta.2015.05.006.

Sánchez, M. L. (2015). ¿La obesidad es una enfermedad? Recuperado de http://marysancheznegro.com/obesidad/

Sasson, M., Lee, M., Jan, C., Fontes, F. y Motta, J. (2014). Prevalence and Associated Factors of Obesity among Panamanian Adults. 1982-2010. PLOS ONE 9(3): e91689. https://doi.org/10.1371/journal.pone.0091689.

Serra-Valdés, M. (2015). Las enfermedades crónicas no transmisibles en la Convención Internacional Cuba-Salud 2015. Rev. Finlay, Cienfuegos, 5(2), 86-88. Recuperado de http://scielo.sld.cu/scielo.php?script=sci_arttext\&p $\mathrm{id}=\mathrm{S} 2221-24342015000200003 \& \operatorname{lng}=\mathrm{es} \& \mathrm{nrm}=\mathrm{iso}$

Spear, B., Barlow, S., Ervin, C., Ludwig, D., Saelens, B., Schetzina, K. y Taveras, E. (2007). Recommendations for treatment of child and adolescent overweight and obesity. Pediatrics, 120(4), 254-88. https://doi.org/10.1542 /peds.2007-2329F

Torrijos, J. M. (2014). Obesidad, el problema que Panamá no sabe resolver. Recuperado de http://laestrella.com.pa/pa nama/nacional/obesidad-problema-panama-sabe-resolver/23808582 
MHSAlud, ISSN: 1659-097X, 17(2), Julio-Diciembre, 2020, PP 1-17

ambulo Arosemena, González Salina, Montenegro, Brooks, Bonilla, Camarena, Moreno

Zamora J. D. y Laclé, A. (2012). Evaluación del gasto energético y actividad física en escolares eutróficos con sobrepeso u obesidad. Revista Chilena de Pediatria, 83(2), 134-145. http://dx.doi.org/10.4067/S0370-4106201200020 0004

CC BY-NC-ND 